\title{
ON PAIRS OF HARMONIC FUNCTIONS
}

R. M. REDHEFFER ${ }^{1}$

Function theory is concerned with pairs of functions $u$ and $v$ which satisfy the Cauchy-Riemann equations,

$$
u_{x}=v_{y}, \quad u_{y}=-v_{x} .
$$

These relations imply that $u$ and $v$ are harmonic, but of course not all harmonic functions satisfy (1). It seems interesting to consider pairs of harmonic functions without the additional restrictions implied in (1), and see to what extent the conjugate character of $u$ and $v$ (over and above their harmonic character) enters into the results of classical analysis. Such is one purpose of the present note. Another purpose is to investigate certain combinations of derivatives introduced by Heinz [1].

The minimun-modulus theorem-To measure conjugateness one may consider the two vectors

$$
A=\left(u_{x}, u_{y}\right), \quad B=\left(v_{x}, v_{y}\right) .
$$

The relations (1) imply

$$
A \perp B, \quad|A|=|B|
$$

and conversely, if (2) holds, we can deduce either (1) or the corresponding relations for functions $f(\bar{z})$. It will not matter to us whether (1) holds or this "reflection" of (1). As a measure of conjugateness, therefore, we introduce

$$
\begin{aligned}
\alpha & =|B|^{2}-|A|^{2}=v_{x}^{2}+v_{y}^{2}-u_{x}^{2}-u_{y}^{2}, \\
\beta & =2 A \cdot B=2\left(u_{x} v_{x}+u_{y} v_{y}\right) .
\end{aligned}
$$

For conjugate functions $\alpha=\beta=0$.

The maximum-modulus theorem of classical function theory asserts that the quantity $u^{2}+v^{2}$ attains its maximum on the boundary, not at an interior point, if (1) holds. Now, it is well known that the same is true for any pair of harmonic functions. (Specifically, we have

$$
\Delta\left(u^{2}+v^{2}\right)=2\left(u_{x}^{2}+u_{y}^{2}+v_{x}^{2}+v_{y}^{2}\right) \geq 0
$$

Presented to the Society, April 28, 1956; received by the editors June 26, 1956.

1 Fellow, National Science Foundation.

This paper was prepared in part under the sponsorship of the Office of Naval Research and the Office of Ordnance Research. 
whenever $\Delta u=\Delta v=0$; and the stated result follows from known properties of subharmonic functions.) Thus it is that in the maximummodulus theorem the conjugate nature of $u$ and $v$ plays no role.

There is also a minimum-modulus theorem, which asserts that if $u^{2}+v^{2}$ has an interior minimum, then the value at the minimum is zero. Here the conjugate character is essential. For example, let $R$ be the square

$$
|x|<\pi / 3, \quad|y|<a, \quad \cosh a=6
$$

and define $u=\cosh y \cos x, v=x$, both harmonic. Then

$$
\begin{aligned}
& u^{2}+v^{2} \geq(\pi / 3)^{2} \text { at the boundary, } \\
& u^{2}+v^{2} \geq 1 / 4 \quad \text { throughout } R
\end{aligned}
$$

but $u^{2}+v^{2}=1$ at the origin. There is, accordingly, a nonzero interior minimum.

The following result is a generalization of the minimum-modulus theorem, to which it reduces when $\alpha=\beta=0$. The condition " $u \geq M$ at the boundary" is to be understood in the sense: $\lim \inf u \geq M$ as $(x, y)$ approaches the boundary through any sequence of interior points.

THEOREM I. Let $u$ and $v$ be harmonic in a bounded region $R$ and let $M$ and $m$ be constants with $M \geq m$. Suppose

$$
\begin{aligned}
& u^{2}+v^{2} \geq M \quad \text { at the boundary, } \\
& u^{2}+v^{2}=m>0, \text { a minimum, at an interior point. }
\end{aligned}
$$

Then, with $\alpha$ and $\beta$ as in (3) we have

$$
\sup \left(\alpha^{2}+\beta^{2}\right)^{1 / 2} \geq \frac{2 m}{D^{2}} \log \frac{M}{m}, \quad(x, y) \in R
$$

where $D$ is the maximum diameter of $R$.

The statement shows clearly how the functions $u$ and $v$ in a given region are required to become "less and less conjugate" as $m$ increases from 0 . Actually we shall prove the stronger result embodied in equation (8) below. The proof depends on the following lemma, which is presented in a form suitable for further applications:

Lemma. Let $h(s)$ be twice differentiable and nondecreasing for inf $\left(u^{2}+v^{2}\right) \leq s \leq \sup \left(u^{2}+v^{2}\right)$. If $u$ and $v$ are harmonic in a bounded region $R$, and satisfy $u^{2}+v^{2} \geq M$ at the boundary, $u^{2}+v^{2}=m$ at an interior point $P$, then 


$$
\sup \left(2 S h^{\prime \prime}+T h^{\prime}\right) \geq 2[h(M)-h(m)] / d^{2}
$$

where $d$ is the maximum distance from $P$ to the boundary of $R$, where the argument of $h^{\prime}$ and $h^{\prime \prime}$ is $u^{2}+v^{2}$, and where

$$
\begin{aligned}
& S=\left(u u_{x}+v v_{x}\right)^{2}+\left(u u_{y}+v v_{y}\right)^{2}, \\
& T=u_{x}^{2}+u_{y}^{2}+v_{x}^{2}+v_{y}^{2} .
\end{aligned}
$$

For proof, take $P$ as origin and define $\rho^{2}=x^{2}+y^{2}$,

$$
2 w=h\left(u^{2}+v^{2}\right)-k \rho^{2}
$$

where $k$ is any constant satisfying

$$
0<k d^{2}<h(M)-h(m) .
$$

Since $\Delta u=\Delta v=0$ a short calculation gives

$$
\Delta w=2 S h^{\prime \prime}(s)+T h^{\prime}(s)-2 k, \quad s=u^{2}+v^{2} .
$$

At $P$ we have

$$
2 w=h(m) .
$$

Also, if $u^{2}+v^{2} \geq M-\epsilon$ on the boundary of a compact subset $R_{n}$ of $R$. then

$$
\begin{aligned}
2 w & \geq h(M-\epsilon)-k d^{2} \\
& =h(M-\epsilon)-h(M)+h(m)+\delta \text { (say) }
\end{aligned}
$$

where by (4) we have $\delta$ fixed and $\delta>0$ for fixed $k$. As $\epsilon \rightarrow 0+$ (and hence for some $R_{n}$ ) we shall have

$$
2 w \geq h(m)+\delta / 2 \text {, on the boundary of } R_{n} .
$$

That being the case, $w$ attains an interior minimum, at which point

$$
2 S h^{\prime \prime}(s)+T h^{\prime}(s) \geq 2 k
$$

by (5). Since $k d^{2}$ is as close as we please to $h(M)-h(m)$, this gives the lemma.

To prove the theorem, choose $h(s)=\log s$ and rewrite $S$ and $T$ in the form

$$
\begin{aligned}
& S=\left(u^{2}+v^{2}\right)\left(u_{x}^{2}+u_{y}^{2}\right)+v^{2} \alpha+u v \beta, \\
& T=2\left(u_{x}^{2}+u_{y}^{2}\right)+\alpha
\end{aligned}
$$

by (3). The conclusion of the lemma is

$$
\sup \left(-2 S / s^{2}+T / s\right) \geq\left(2 / d^{2}\right) \log (M / m) .
$$

Now, in the notation (6), 


$$
\begin{aligned}
\left|-2 S / s^{2}+T / s\right| & =\left|\left(1 / s^{2}\right)\left(\alpha s-2 v^{2} \alpha-2 u v \beta\right)\right| \\
& =\left|\left(1 / s^{2}\right)\left[\alpha\left(u^{2}-v^{2}\right)-2 u v \beta\right]\right| \\
& \leqq\left(1 / s^{2}\right)\left(\alpha^{2}+\beta^{2}\right)^{1 / 2} s
\end{aligned}
$$

by the Schwarz inequality.

Hence (7) gives

$$
\sup \frac{\left(\alpha^{2}+\beta^{2}\right)^{1 / 2}}{u^{2}+v^{2}} \geq\left(2 / d^{2}\right) \log (M / m)
$$

which is a result stronger than that of the theorem. The latter is found by putting the minimum of $u^{2}+v^{2}$, namely $m$, on the left side of (8) and by putting $D$, the maximum of $d$, on the right.

An inequality for the derivatives. In the following result the measures of conjugateness, $\alpha$ and $\beta$, do not appear. It will be indicated in the next section that (at least for simple regions) the conjugate character of $u$ and $v$ is truly irrelevant:

THEOREM II. ${ }^{2}$ Let $u$ and $v$ be harmonic in a bounded region $R$ and let $M$ and $m$ be constant. Suppose

$$
\begin{aligned}
& u^{2}+v^{2} \geq M \text { at the boundary, } \\
& u^{2}+v^{2}=m \text { at the interior point, } P .
\end{aligned}
$$

If $d$ is the maximum distance from $P$ to the boundary of $R$, then

$$
\sup \left(u_{x}^{2}+u_{y}^{2}+v_{x}^{2}+v_{y}^{2}\right) \geq 2(M-m) / d^{2}, \quad(x, y) \in R .
$$

The proof is immediate if we simply choose $h$ linear in the lemma, but we wish to show in what sense that choice is optimum. By Schwarz's inequality, in the previous notation,

$$
S \leqq\left(u^{2}+v^{2}\right) T=s T
$$

and hence the lemma yields

$$
\sup T\left(2 s h^{\prime \prime}+h^{\prime}\right) \geq 2[h(M)-h(m)] / d^{2}
$$

whenever $h^{\prime \prime} \geq 0$. This shows that at some interior point

$$
T \geq \frac{2}{d^{2}} \frac{h(M)-h(m)}{2 s h^{\prime \prime}(s)+h^{\prime}(s)}-\epsilon,
$$

The intent is so to choose $h(s)$ that (10) is as strong as possible, whence we are led to consider

2 Compare [1, Satz 1.] 


$$
\sup _{h} \inf _{s} \frac{h(M)-h(m)}{2 s h^{\prime \prime}(s)+h^{\prime}(s)}, \quad h^{\prime \prime} \geq 0, h^{\prime} \geq 0 .
$$

By the mean-value theorem,

$$
\frac{h(M)-h(m)}{2 s h^{\prime \prime}(s)+h^{\prime}(s)}=\frac{h^{\prime}(\xi)(M-m)}{2 s h^{\prime \prime}(s)+h^{\prime}(s)} \leqq \frac{h^{\prime}(\xi)}{h^{\prime}(s)}(M-m) .
$$

Evidently, the inf is $\leq M-m$. On the other hand the inf is attained when $f(s) \equiv s$; and we have established that

$$
\sup _{h} \inf _{s} \frac{h(M)-h(m)}{2 s h^{\prime \prime}(s)+h^{\prime}(s)}=M-m .
$$

This yields Theorem II, and shows that the simple choice $h(s)=s$ introduces no wastefulness over and above that resulting from our use of (9).

Analysis of the foregoing result. In connection with Theorem II three questions naturally present themselves: Is the result optimum for harmonic functions? Is a stronger result valid for conjugate functions? Is the result false if the functions are neither harmonic nor conjugate? We shall give complete answers to these questions in a special case.

THEOREM III. Let $u$ and $v$ be differentiable in a circle $R$ of radius $r$. Suppose

$$
\begin{aligned}
& u^{2}+v^{2} \geq M \text { at the boundary, } \\
& u^{2}+v^{2}=0 \text { at the center. }
\end{aligned}
$$

Then the value of

$$
\inf _{(u, v)} \sup _{(x, y)}\left(u_{x}^{2}+u_{y}^{2}+v_{x}^{2}+v_{y}^{2}\right)
$$

is $M / r^{2}$ when $u$ and $v$ are unrestricted; it is $2 M / r^{2}$ when $u$ and $v$ are harmonic: and it is $2 M / r^{2}$ when $u$ and $v$ are conjugate harmonic.

Hence in this special case Theorem II is optimum; it is false, but a weaker result is true, for nonharmonic functions; and it cannot be improved if we impose conjugateness as an additional hypothesis.

For proof of the first statement let $\epsilon$ be constant and define

$$
w^{2}=u^{2}+v^{2}+\epsilon, \quad 0<\epsilon<M,
$$

so that $w$ is differentiable. At some point $a$ on the segment $(0 \leq x<r$, $y=0$ ) we have $w^{2}=M$, in view of the condition $u^{2}+v^{2} \geq M$ at the boundary. By the theorem of the mean 


$$
\begin{aligned}
& M^{1 / 2}-\epsilon^{1 / 2}=w(a, 0)-w(0,0) \\
& =a w_{x}(\xi, 0) \quad[0<\xi<a] \\
& =a\left(u u_{x}+v v_{x}\right) / w \quad[\text { at }(\xi, 0)] \\
& \leqq a\left(u_{x}^{2}+v_{x}^{2}\right)^{1 / 2}\left(u^{2}+v^{2}\right)^{1 / 2} / w \quad \text { [Schwarz } 1 \\
& <r\left(u_{x}^{2}+v_{x}^{2}\right)^{1 / 2}
\end{aligned}
$$

Since $\epsilon$ is arbitrary we have shown

$$
\sup \left(u_{x}^{2}+v_{x}^{2}\right) \geq M / r
$$

so that surely

$$
\sup \left(u_{x}^{2}+u_{y}^{2}+v_{x}^{2}+v_{y}^{2}\right) \geq M / r^{2} .
$$

On the other hand the choice $v=0$ and

$$
\left\{\begin{array}{l}
u=k\left(\rho^{2}+\epsilon^{2}\right)^{1 / 2}-k \epsilon, \\
\left.k=M^{1 / 2} /\left[\left(r^{2}+\epsilon^{2}\right)^{1 / 2}-\epsilon\right)\right], \\
\epsilon>0, \text { constant }
\end{array}\right.
$$

gives

$$
\begin{cases}u^{2}+v^{2}=0 & \text { at the origin, } \\ u^{2}+v^{2}=M & \text { at the boundary, } \\ u^{2}+v^{2} & \text { twice differentiable. }\end{cases}
$$

Also we have

$$
\begin{aligned}
u_{x} & =k \rho \rho_{x} /\left(\rho^{2}+\epsilon^{2}\right)^{1 / 2} \\
& =k x /\left(\rho^{2}+\epsilon^{2}\right)^{1 / 2}
\end{aligned}
$$

so that, by symmetry,

$$
u_{x}^{2}+u_{y}^{2}=k^{2} \rho^{2} /\left(\rho^{2}+\epsilon^{2}\right) .
$$

Hence,

$$
\begin{aligned}
\sup \left(u_{x}^{2}+u_{y}^{2}\right) & =k^{2} r^{2} /\left(r^{2}+\epsilon^{2}\right) \\
& =M \frac{r^{2}}{\left(r^{2}+\epsilon^{2}\right)\left(r^{2}+\epsilon^{2}\right)^{1 / 2}-\epsilon^{2}} \\
& \rightarrow M / r^{2} \text { as } \epsilon \rightarrow 0+.
\end{aligned}
$$

This yields the desired result. It is interesting that the inf is never attained (we did not really prove that, but it seems clear). Also, one 
can do as well with $v=0$ as with $v$ arbitrary. And finally, the condition $u^{2}+v^{2} \geq M$ at the boundary could be replaced by $u^{2}+v^{2} \geq M$ at one boundary-point.

Turning to the part of the theorem involving harmonic functions, we see from Theorem II that

$$
\sup \left(u_{x}^{2}+u_{y}^{2}+v_{x}^{2}+v_{y}^{2}\right) \geq 2 M / r^{2}
$$

since the value $m=0$ is attained at the origin, and the distance from there to any boundary point is $r$. On the other hand the choice

$$
u=M^{1 / 2}(x / r), \quad v=M^{1 / 2}(y / r)
$$

gives equality, so that the second part of the result is established. Since $u$ and $v$ happen to be conjugate functions, the third statement is also verified. Here the inf is actually attained.

The foregoing results may be expressed in terms of the mapping $R \rightarrow R^{*}$ which takes the origin into the origin and is given by the harmonic functions

$$
u=u(x, y), \quad v=v(x, y) .
$$

Let $S$ be a subset of $R$ and $S^{*}$ the image of $S$. Suppose $S$ is contained in the circle $\rho \leq r$ while $S^{*}$ contains the circle $\rho<r^{*}$. Then we have

$$
\sup \left(u_{x}^{2}+u_{y}^{2}+v_{x}^{2}+v_{y}^{2}\right) \geq 2\left(r^{*} / r\right)^{2}, \quad(x, y) \in S .
$$

Since $u$ and $v$ are harmonic, so are their derivatives; and hence

$$
T=u_{x}^{2}+u_{y}^{2}+v_{x}^{2}+v_{y}^{2}
$$

is subharmonic, $\Delta T \geq 0$. The sup of $T$ is attained, therefore, on the boundary of $S$, and we have a third formulation

$$
\frac{\sup \left(u_{x}^{2}+u_{y}^{2}+v_{x}^{2}+v_{y}^{2}\right) \sup \left(x^{2}+y^{2}\right)}{\inf \left(u^{2}+v^{2}\right)} \geq 2 .
$$

Here the sup and inf are taken over the boundary of any compact set $S \subset R$ which contains the origin in its interior. The choice $u=x, v=y$ gives equality when $S$ is a circle centered at the origin.

Characteristic values. It is our intention to obtain a bound in the opposite direction; that is, to estimate

$$
\inf \left(u_{x}^{2}+u_{y}^{2}+v_{x}^{2}+v_{y}^{2}\right)
$$

from above. To this end, we recapitulate a few elementary facts con- 
cerning the fundamental mode of a membrane.

A characteristic value for a region $R$ is a number, $k$, such that the boundary-value problem

$$
\begin{aligned}
\Delta u+k u & =0 \text { in } R, \\
u & =0 \text { at the boundary }
\end{aligned}
$$

has a nontrivial solution, $u$. If $f$ is any twice-differentiable function positive in $R$ and on the boundary, it is well known $[2 ; 3]$ that every such $k$ satisfies

$$
k \geq \inf \left\{-\frac{\Delta f}{f}\right\}, \quad(x, y) \in R .
$$

With $\epsilon>0$, a constant, the choice

$$
f=r^{2}+\epsilon-u^{2}-v^{2}
$$

leads at once to the following, which supplements Theorem II:

THEOREM IV. Let $u$ and $v$ be harmonic functions which map a region $R$ into a region $R^{*}$ contained in a circle of radius $r$. Then

$$
\inf \left(u_{x}^{2}+u_{y}^{2}+v_{x}^{2}+v_{y}^{2}\right) \leq k r^{2} / 2
$$

when $k$ is the smallest characteristic value for $R$.

We remark, in conclusion, that (11) yields an inequality for $\beta$ alone, supplementary to Theorem I. Namely, if $u v$ has a positive lower bound in $R$, and if $u$ and $v$ are harmonic, then

$$
\inf [-\beta /(u v)] \leq k, \quad(x, y) \in R .
$$

\section{BIBLIOGRAPHY}

1. E. Heinz, Über die Lösungen der Minimalfächengleichung, Nachr. Ges. Wiss. Göttingen vol. 8 (1952) pp. 51-56.

2. J. Barta, Sur la vibration fondamentale d'une membrane, C. R. Acad. Sci. Paris vol. 204 (1937) pp. 472-473.

3. R. J. Duffin, Lower bounds for eigenvalues, The Physical Review vol. 71 (1947) pp. 827-828.

University of California, Los Angeles 